\title{
Chemistry of the American cockroach pheromone
}

\author{
Kenji Mori*, Shigefumi Kuwahara, and Yasuhiro Igarashi \\ Department of Agricultural Chemistry, The University of Tokyo, \\ Yayoi 1-1-1, Bunkyo-ku, Tokyo 113, Japan
}

\begin{abstract}
Abstroct - (-)-Periplanone-B (1), Hauptmann's (-)-periplanone-A (7), and the racemate 27 of the rearrangement product (PA 22-VII) of Persoons's periplanone-A were synthesized. Persoons's periplanone-A was shown to be the thermal decomposition product $\mathbf{8}$ of Hauptmann's periplanone-A, and was biologically inactive.
\end{abstract}

\section{INTRODUCTION}

In 1974 Persoons of al. isolated two highly active sex pheromone components of the American cockroach (Periplaneto americano), ${ }^{1}$ and named them periplanone-A and periplanone-B. ${ }^{2}$ The proposed structure 1 of the latter ${ }^{3,4}$ was confirmed by numerous syntheses, either as the racemate ${ }^{5-9}$ or as the naturally occurring enantiomer (-)-1 (Fig. 1). ${ }^{10-13}$ The structure of the labile periplanone-A, however, has remained obscure. The original structural proposal of periplanone-A as 2 by Persoons of ol..$^{14,15}$ was challenged by Shizuri of al. They synthesized two hydroazulenones $( \pm)-3^{16}$ and $( \pm)-4,{ }^{17}$ both of which showed the spectra different from those of the pheromone isolated by Persoons. ${ }^{15}$ Macdonald of al. proposed periplanone-A to have structure 5 , based on their synthesis of $( \pm)-5$ which was thought to be bioactive. ${ }^{18}$ The structure of the pheromone isolated by Persoons was postulated by them as 6 , the possible transannular cyclization product of 5.18 However, pure synthetic $( \pm)-5$ later proved to be only weakly bioactive, and therefore 5 could not be periplanone-A. ${ }^{19,20}$

Meanwhile, Hauptmann $\theta f$ al. isolated 7 from the American cockroach, named it periplanone-A, synthesized ( \pm )-7, and found it to be bioactive. ${ }^{21}$ Subsequently, the same compound 7 was also isolated by Nishino ef al. together with periplanone-B (1), and 7 was again called periplanone-A.22 Although synthetic ( \pm )-7 was highly bioactive, ${ }^{19-21}$ the spectral data of 7 were quite different from those reported for periplanone-A by Persoons. ${ }^{15}$ Hauptmann's periplanone-A is therefore not identical with Persoons's periplanone-A. It should be added that neither Hauptmann nor Nishino was able to reisolate Persoons's periplanone-A from the cockroach.

To account for the ${ }^{1} \mathrm{H}$ NMR spectral data of Persoons's periplanone-A and also on the basis of molecular mechanics calculations, Shizuri of al. proposed 8 as the plausible structure of Persoons's periplanone-A ${ }^{23}$ However, they recently reported that their synthetic $( \pm)-8$ showed an NMR spectrum different from that of Persoons's periplanone-A. ${ }^{24}$ In short, there is no conclusive work to clarify the structure of Persoons's periplanone-A.

We became interested in this problem, and derived a strategy to solve the problem: (i) An efficient enantioselective synthesis of Hauptmann's periplanone-A [(-)-7] was to be developed, because this would allow investigation of the reactivity of 7 . Prior to the synthesis of $(-)-7$, it would be desirable to achieve a more efficient synthesis of ( - )-periplanone-B (1), because our previous synthesis starting from (+)-dihydrolimonene furnished (-)-1 in only $0.5 \%$ overall yield. ${ }^{11,12}$ The new synthesis of (-)-1 should be flexible enough to be modified for the synthesis of (-)-7. (ii) Because Persoons's periplanone-A was reported to be labile, its stable rearrangement product (27, vide infro) should be synthesized so as to verify (at least a part of) Persoons's structural work. (iii) Assuming that Hauptmann's periplanone-A (7) is the genuine pheromone, its possible conversion to Persoons's periplanone-A must be attempted. Our work along this line finally enabled us to give unambiguously the structure 8 to Persoons's periplanone-A, which was biologically inactive.
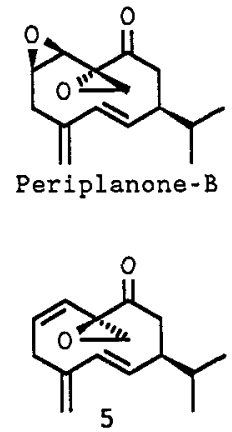
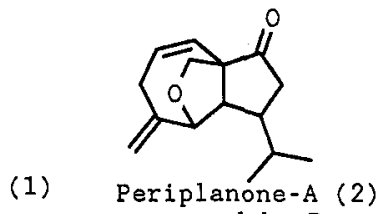

as proposed by Persoons

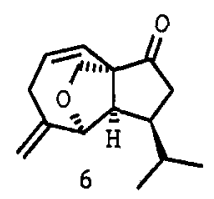

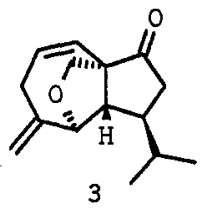

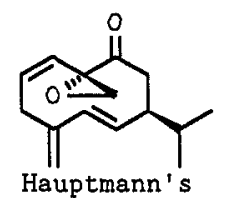

periplanone-A (7)
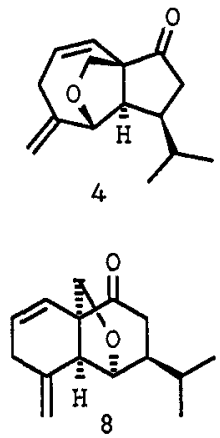

Fig. 1. Periplanone-B and Periplanone-A. 


\section{SYNTHESIS OF (-)-PERIPLANONE-B}

Fig. 2 shows our new enantioselective and efficient synthesis of (-)-periplanone-B (1). ${ }^{13}$ Starting from (S)-3-cyclohexene-1-carboxylic acid (9), $(S)-11$ was obtained in $86 \%$ overall yield, which was the optically active version of the intermediate used by Still. ${ }^{5}$ Like him, we also utilized the oxy-Cope rearrangement as one of the key-steps. ${ }^{5}$ We employed, however, 14 as the substrate for the anionic oxy-Cope rearrangement, because it would yield 15, the optically active and cis-isomer of Still's intermediate. The racemate of the enone ( \pm )-15 (TBDPS instead of EE) was shown, in Takahashi's synthesis of $( \pm)-1$, to give the desired $\beta$-epoxide exclusively, when treated with $A-B u O O K^{8}$ According to the previous synthesis, ${ }^{11,12}$ our optically active 16 was converted to $(-)$-periplanone-B (1), m.p. 55.5-57.5 $5^{\circ},[\alpha]_{D}^{21}-552^{\circ}$ ( $n$-hexane). The present 18-step-synthesis of $(-)-1$ was accomplished in $11 \%$ overall yield to give about $300 \mathrm{mg}$ of $(-)-1$. This is the highest overall yield that ever has been reported.

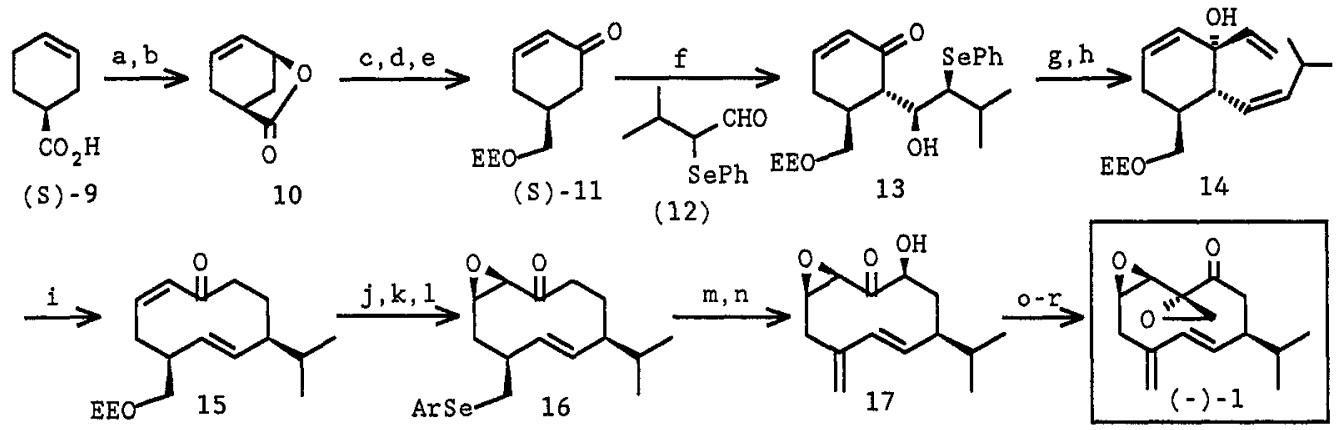

Reagents: a) $\mathrm{KI}_{3}, \mathrm{NaHCO}_{3}$ aq; b) $\mathrm{DBU}$; c) $\mathrm{LAH}$; d) $\mathrm{MnO}_{2}$ [86\% from (S)-9]; e) ethyl vinyl ether, PPTS $(99 \%) ;$ f) LDA, $-78^{\circ} \mathrm{C}(20 \mathrm{~min}),-15^{\circ} \mathrm{C}$ (3 min); g) $\mathrm{MsCl}, \mathrm{Et}_{3} \mathrm{~N}\left(51 \%\right.$ from 11); h) $\mathrm{CH}_{2}=\mathrm{CHLi}(86 \%)$ i)

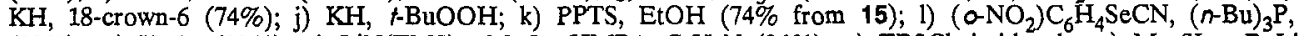
(99\%); m) $\mathrm{H}_{2} \mathrm{O}_{2}(90 \%)$; n) LiN(TMS) ${ }_{2}, \mathrm{MoO}_{5} \cdot \mathrm{HMPA} \cdot \mathrm{C}_{5} \mathrm{H}_{5} \mathrm{~N}(86 \%)$; o) TBSCl, imidazole; p) $\mathrm{Me}_{3} \mathrm{SI}$, $n$-BuLi; q) $\left.(n-B u)_{4} \mathrm{NF} ; \mathrm{r}\right)$ PDC (68\% from 17).

Fig. 2. Synthesis of (-)-periplanone-B.

\section{SYNTHESIS OF HAUPTMANN'S (-)-PERIPLANONE-A}

We then turned our attention to the synthesis of Hauptmann's (-)-periplanone-A (7). The synthesis must be efficient enough to furnish over $100 \mathrm{mg}$ of 7 so that we can examine its stability and reactivity. Our synthetic scheme is illustrated in Fig. 3.25 The optically active version 22 of Still's trienone intermediate served as our intermediate, which was prepared from $(R)-9$, the antipode of the acid employed in the synthesis of $(-)$-periplanone-B (Fig. 2$)$. The acid $(R)-9$ was converted to $(R)-11$ in the same manner as shown in Fig. 2. The zinc enolate derived from 11 was condensed with 12 to give, after elimination followed by addition of vinyllithium, a separable mixture of $19(43 \%)$ and 20 (17\%). The anionic oxy-Cope rearrangement of 19 furnished 21 in $80 \%$ yield. Hydroxylation of 21 was followed by protection-deprotection of the hydroxyl groups and elimination of one of them via selenide to give the key trienone 22. Treatment of 22 with chloromethyllithium followed by deprotection of the silyl protective group gave 23, which was oxidized to give two isomeric ketones, 5 and 7 . The major isomer obtained in $41 \%$ yield from 22 was $(+)-5,[\alpha]_{D}^{25}+182^{\circ}(n$-hexane), which had been proposed to be periplanone-A by Macdonald.$^{18}$ Hauptmann's periplanone-A $[(-)-7]$, m.p. $42-44^{\circ} \mathrm{C},[\alpha]_{\mathrm{D}}^{24}-574^{\circ}$ (n-hexane), was the minor isomer (24\% yield from 22). Its structure 7 was confirmed by an X-ray crystallographic analysis. The overall yield of (-)-7 from 9 was $2.1 \%$ through 18 steps, and about $230 \mathrm{mg}$ of (-)-7 became available. Pheromone activity of our synthetic samples was evaluated by observing the wing-raising behavior of the male American cockroaches. $(-)$-Periplanone-B (1) was active at $10^{-7} \mu \mathrm{g}$, and Hauptmann's (-)-periplanone-A (7) showed the activity at $10^{-5} \mu \mathrm{g}$, while $(+)-5$ was active at such a big dose of $10^{-1} \mu \mathrm{g}$.
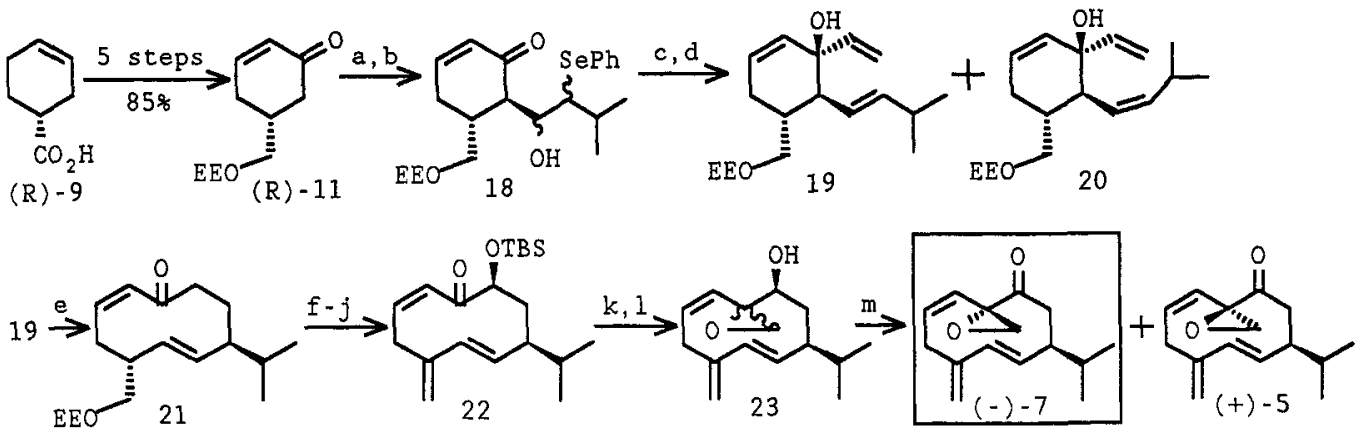

Reagents: a) LDA, TMSCl (98\%); b) $\mathrm{MeLi}, \mathrm{ZnCl}_{2}, 12$; c) $\mathrm{MsCl}_{1} \mathrm{Et}_{3} \mathrm{~N}$ (89\% from 11); d) $\mathrm{CH}_{2}=\mathrm{CHLi}(43 \%$ and $17 \%)$; e) $\mathrm{KH}, 18$-crown-6 (80\%); f) LiN(TMS), $\mathrm{MoO}_{5} \cdot \mathrm{HMPA} \cdot \mathrm{C}_{5} \mathrm{H}_{5} \mathrm{~N}(86 \%) ; \mathrm{g}$ ) TBSCl, imidazole; h) PPTS, EtOH; i) $\left.a-\left(\mathrm{NO}_{2}\right) \mathrm{C}_{6} \mathrm{H}_{4} \mathrm{SeCN},(n-\mathrm{Bu})_{3} \mathrm{P} ; \mathrm{j}\right) 35 \% \mathrm{H}_{2} \mathrm{O}_{2}$ (40\% from 21); k) $\left.\mathrm{ICH}_{2} \mathrm{Cl}, \mathrm{MeLi} ; \mathrm{l}\right)(n-\mathrm{Bu})_{4} \mathrm{NF}$; m) $\mathrm{CrO}_{3} \cdot 2 \mathrm{C}_{5} \mathrm{H}_{5} \mathrm{~N}$ (24\% and $41 \%$ from 22).

Fig. 3. Synthesis of Hauptmann's (-)-periplanone-A. 


\section{SYNTHESIS OF THE RACEMATE OF PA 22-VII}

In 1978 Persoons of al. reported the labile nature of their periplanone-A to give a stable and biologically inactive rearrangement product with a code name PA 22-VII. ${ }^{14}$ Indeed, about $50 \%$ conversion of their periplanone-A to PA 22-VII was observed at $0^{\circ} \mathrm{C}$ in two weeks, ${ }^{14}$ and even at $-20^{\circ} \mathrm{C}$ the rearrangement took place. ${ }^{15}$ The rearrangement product was thought to be $24,{ }^{14}$ to which the stereostructure 25 was later assigned. ${ }^{15}$ Macdonald's synthesis of $( \pm)-26$ was followed by comparison of its ${ }^{1} \mathrm{H}$ NMR spectrum with that reported for PA 22 -VII. 18 This enabled Macdonald of al. to propose 27 as the structure of the rearrangement product, PA 22-VII. ${ }^{18}$ The same conclusion was also announced by Shizuri of ol. ${ }^{23}$ In order to confirm this proposal, we synthesized ( \pm )-27 as shown in Fig. $4 .^{26}$

Our synthesis started from the known Diels-Alder adduct $\left( \pm\right.$ )-28 (cis:trans=1:1.2). Reduction of 28 with $\mathrm{LiAl}(\mathrm{OH}-\mathrm{Bu})_{3} \mathrm{H}$ yielded crystalline $( \pm)-29$, which was converted to $( \pm)-30$. Mesylation of 30 was followed by deprotection, base treatment, and oxidation to give $( \pm)-31$. This was submitted to the conjugate addition to furnish $( \pm)-32$, the structure of which was confirmed by an X-ray crystallographic analysis. Reduction of 32 with $\mathrm{Li} / \mathrm{NH}_{3}$ was followed by silylation and bromination to give $( \pm)-33$, which was converted to the diene $( \pm)-34$. Photosensitized oxygenation of 34 followed by LAH reduction and $\mathrm{MnO}_{2}$ oxidation afforded $( \pm)-35$. Hydrogenation of 35 was followed by its olefination to give $( \pm)-36$. Dehydration of 36 gave $( \pm)$-37. After its deprotection, the resulting alcohol was oxidized to give ( \pm )-27, m.p. $118.0-118.5^{\circ} \mathrm{C}$, whose ${ }^{1} \mathrm{H}$ NMR and MS data were identical with those reported for Persoons's rearrangement product, PA 22-VII. ${ }^{14,15}$ The structural proposal for PA 22-VII was thus confirmed. Then, what is Persoons's periplanone-A?
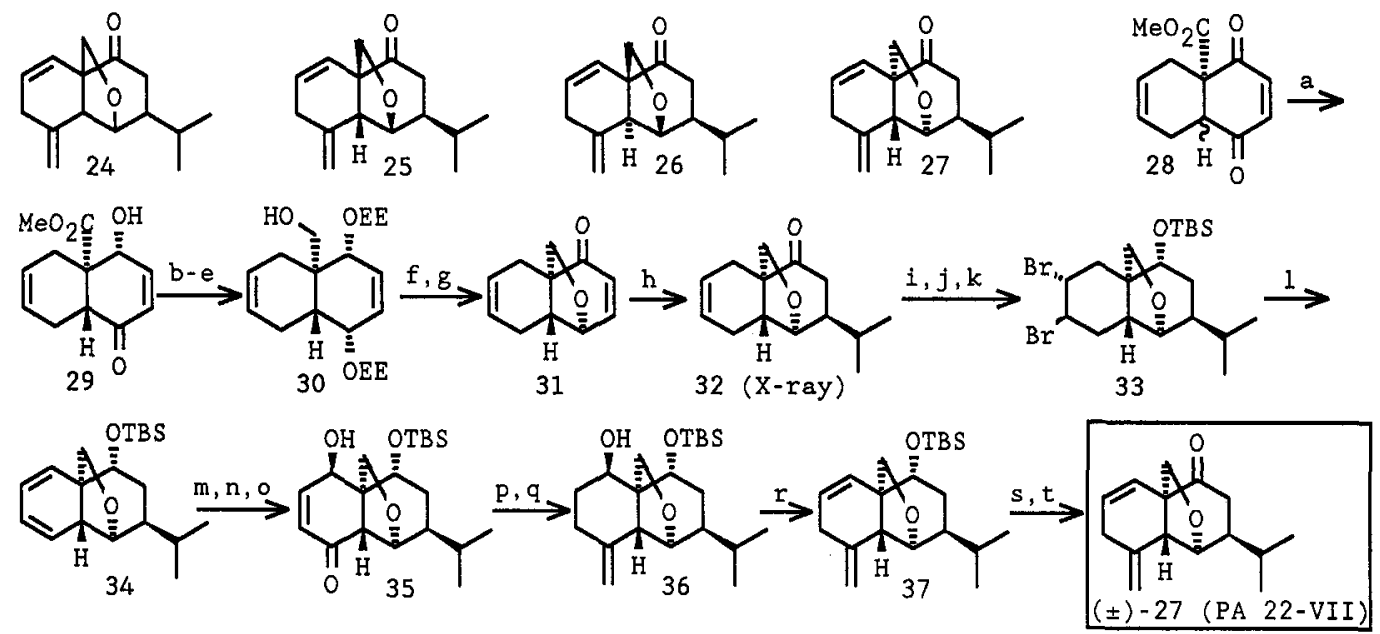

Reagents: a) i) $\mathrm{LiAl}(\mathrm{O} t-\mathrm{Bu})_{3} \mathrm{H}, 0 \sim 5^{\circ} \mathrm{C}$; ii) chromatog. (39\%); b) ethyl vinyl ether, PPTS (quant.); c) $\mathrm{NaBH}_{4}$, $\mathrm{CeCl}_{3}(71 \%)$; d) ethyl vinyl ether, PPTS (77\%); e) LAH (87\%); f) i) $\mathrm{MsCl}, \mathrm{C}_{5} \mathrm{H}_{5} \mathrm{~N}$, DMAP; ii) MeOH, PPTS; iii) $2.5 \%$ NaOMe in MeOH (81\% in 3 steps); g) ( $\mathrm{COCl})_{2}$, DMSO, $\mathrm{Et}_{3} \mathrm{~N}(92 \%) ; \mathrm{h}$ ) $\quad$ PrMgI, CuCN (75\%); i) $\mathrm{Li}, \mathrm{NH}_{3}$, THF, 4 -BuOH (91\%); j) TBSOTf, 2,6-lutidine (quant.); k) $\mathrm{C}_{5} \mathrm{H}_{5} \mathrm{~N} \cdot \mathrm{HBr} \cdot \mathrm{Br}_{2}$ (quant.); l) DBU; m) $\mathrm{O}_{2}$ $h \nu$, Methylene Blue; n) $\mathrm{LAH},\left(15 \%\right.$ in 3 steps); o) $\mathrm{MnO}_{2}$ (83\%); p) $\mathrm{H}_{2}, 10 \% \mathrm{Pd} / \mathrm{C}$ (99\%); q) i) $\mathrm{Ac}_{2} \mathrm{O}$, $\mathrm{C}_{5} \mathrm{H}_{5} \mathrm{~N}$ (98\%); ii) $\mathrm{Ph}_{3} \mathrm{PMeBr}, n-\mathrm{BuLi}(56 \%)$; iii) MeLi 94\%); r) i) TfCl, DMAP; ii) DBU, $70^{\circ} \mathrm{C}$ (78\% in 2 steps); s) (n-Bu) ${ }_{4} \mathrm{NF}$ (quant.); t) PDC, $\mathrm{CH}_{2} \mathrm{Cl}_{2}$, MS $3 \mathrm{~A}(81 \%)$.

Fig. 4. Synthesis of the rearrangement product ( \pm )-PA 22-VII.

\section{CLARIFICATION OF THE STRUCTURE OF PERSOONS'S PERIPLANONE-A}

We carefully read the previous reports on the isolation of periplanone-A and $B, 14,14,15,21,22$ including Persoons's doctoral dissertation. ${ }^{27}$ Persoons of al. purified periplanone-A finally by GLC, $14,15,27$ while Hauptmann of al. did not expose their samples to such high temperatures. ${ }^{21}$ This suggested that Persoons's periplanone-A was a thermal rearrangement product of Hauptmann's periplanone-A. The same speculation was made by Nishino $\theta t a l^{22}$ and also by Hauptmann. ${ }^{28}$ The first attempt to solve the problem was the GC-MS analysis of our synthetic Hauptmann's periplanone-A [(-)-7] using a column packed with $3 \%$ OV-17 at $180^{\circ} \mathrm{C} .{ }^{29}$ The gas chromatogram showed mainly two peaks. The mass spectrum of the major peak was due to (-)-7 itself. The minor peak with a longer retention time gave the MS which was very similar to that published for Persoons's periplanone-A. ${ }^{14,15}$ In order to isolate the decomposition product, the thermolysis of a total amount of $80 \mathrm{mg}$ of (-)-7 was carried out by GLC (column, $3 \%$ OV-17, $2 \mathrm{~m}$ x $6 \mathrm{~mm}$ at $220^{\circ} \mathrm{C}$; injection $260^{\circ} \mathrm{C}$; carrier gas, $\mathrm{N}_{2}, 45 \mathrm{ml} / \mathrm{min}$ ). Under these conditions, the decomposition was achieved quite efficiently. After TLC purification, the major decomposition product was isolated in $71 \%$ yield. Its ${ }^{1} \mathrm{H}$ NMR spectrum was identical with the published spectrum of Persoons's periplanone-A, although Persoons's sample was contaminated with PA 22-VII (27) . $^{14,15}$ Furthermore, its IR spectrum was exactly the same as that published for Persoons's periplanone-A. ${ }^{27}$ Therefore, the major product obtained by the thermolysis of Hauptmann's periplanone-A must surely be Persoons's periplanone-A.

The next task was the structure elucidation of the thermolysis product (Fig. 5). Upon reduction with $\mathrm{NaBH}_{4}$, it gave a crystalline alcohol, m.p. $77-78^{\circ} \mathrm{C}$, the structure of which was determined to be 38 by an X-ray crystallographic analysis (see Fig. 6 for the ORTEP diagram). The Swern oxidation of the pure alcohol 38 regenerated the thermolysis product (= Persoons's periplanone-A), $[\alpha]_{D}^{25}-290^{\circ}$ ( $n$-hexane). Accordingly, the structure of Persoons's periplanone-A was finally established as 8 . This is the structure previously proposed by Shizuri of al..$^{23}$ Shizuri's structural analysis by means of modern calculations was therefore correct. As mentioned earlier, however, a synthesis of $( \pm)-8$ was recently reported by Shizuri of ol. with the claim that their $( \pm)-8$ showed the ${ }^{1} \mathrm{H}$ NMR spectrum different from that of Persoons's periplanone-A. ${ }^{24}$ Because of the unambiguous nature of our structure determination of the thermolysis product, Shizuri's result must have been due to an ambiguity in the course of their synthesis to afford presumably an isomer of $( \pm)-8$. 


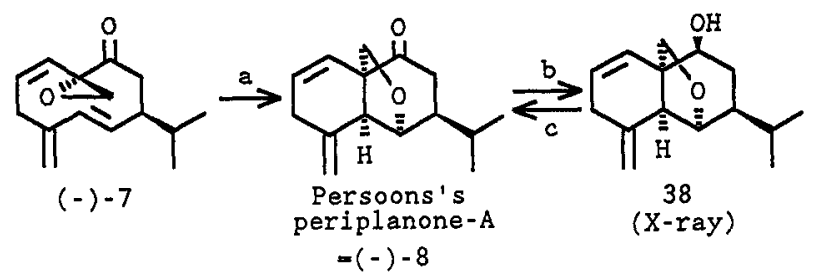

Reagents: a) $220^{\circ} \mathrm{C}(71 \%)$; b) $\mathrm{NaBH}_{4}, \mathrm{MeOH},-10^{\circ} \mathrm{C}, 3 \min (80 \%)$; c) DMSO, $(\mathrm{COCl})_{2}, \mathrm{Et}_{3} \mathrm{~N}, \mathrm{CH}_{2} \mathrm{Cl}_{2},-60^{\circ} \mathrm{C} \sim$ r.t., $15 \min (87 \%)$.

Fig. 5. Structure elucidation of Persoons's periplanone-A.

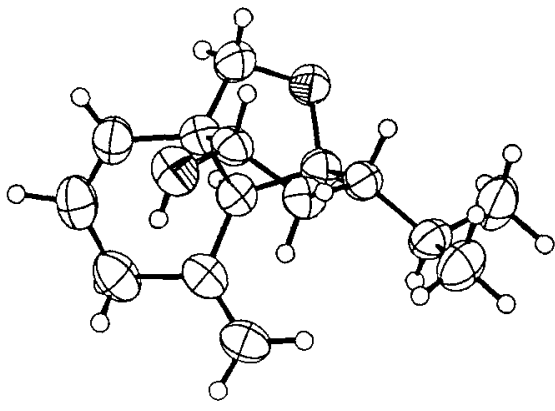

Fig. 6. $X$-ray structure of $\mathbf{3 8 .}$

To complete our study on $(-)-8$, we then examined its pheromone activity. Persoons's periplanone-A [(-)-8] obtained by TLC purification of the crude thermolysis products was bioactive at $10^{-3} \mu \mathrm{g}$ as reported by Persoons. However, pure $(-)-8$ obtained by the Swern oxidation of pure crystalline 38 was biologically inactive even at $10 \mu \mathrm{g}$. The activity of the crude ( -$) \cdot 8$ must have been due to the contamination of the intact Hauptmann's periplanone-A [(-)-7]. It must be added that even the crude (-)-8 was seemingly quite pure as judged by its $300 \mathrm{MHz}{ }^{1} \mathrm{H}$ NMR spectrum. The bioassay was far more sensitive in detecting the contaminating (-) -7. The pheromone activity of $(-)-8$ as reported by Persoons of al..$^{14}$ therefore seems to be ascribable to the contamination of a minute amount of $(-)-7$ and/or $(-)-1$. As to the stability of $(-)-8$, it was quite stable contrary to Persoons's observation that 8 was unstable and gradually changed into PA 22-VII (27). ${ }^{14}$ Some unknown impurities in their sample must have accelerated this conversion.

\section{CONCLUSION}

In summary, Persoons's periplanone-A was an artifact derived from Hauptmann's periplanone-A, and devoid of pheromone activity. A lesson learned through whole of the works related to Persoons's periplanone-A is that one should employ as mild conditions as possible in isolating an unstable and extremely bioactive compound to avoid possible decomposition and confusion. Production of an artifact may demand tremendous amount of rewardless efforts by others to restore the proper order out of the chaos.

\section{REFERENCES}

1. C. J. Persoons, F. J. Ritter, and W. J. Richtendonk, Proc. Kon. Ned. Acad. Wetensch., C77, 201 (1974).

2. F. J. Ritter and C. J. Persoons, Neth. J. Zool, 25, 261 (1975).

3. C. J. Persoons, P. E. J. Verwiel, F. J. Ritter, E. Talman, P. J. F. Nooijen, and W. J. Nooijen, Tetrahedron Lett., 2055 (1976).

4. C. J. Persoons, P. E. J. Verwiel, E. Talman, and F. J. Ritter, J. Chem. Ecol., 5, 221, (1979).

5. W. C. Still, J. Am. Chem. Soc., 101, 2493 (1979).

6. S. L. Schreiber and C. Santini, J. Am. Chem. Soc., 106, 4038 (1984).

7. H. Hauptmann, G. Muhlbauer, and M. P. C. Walker, Tetrahedron Letf., 27, 1315 (1986).

8. T. Takahashi, Y. Kanda, H. Nemoto, K. Kitamura, J. Tsuji, and Y. Fukazawa, J. Org. Chem., 51, 3393 (1986).

9. S. G. Cauwberghs and P. J. DeClercq, Tetrahedron Letf., 29, 6501 (1988).

10. M. A. Adams, K. Nakanishi, W. C. Still, E. V. Arnold, J, Clardy, and C. J, Persoons, J. Am. Chem. Soc., 101, 2495 (1979).

11. T. Kitahara, M. Mori, K. Koseki, and K. Mori, retrohedron Letf., 27, 1343 (1986).

12. T. Kitahara, M. Mori, and K. Mori, Tetrahedron, 43, 2689 (1987).

13. S. Kuwahara and K. Mori, Heterocycles, 28, 167 (1989).

14. E. Talman, P. E. J. Verwiel, F. J. Ritter, and C. J. Persoons, Isr. J. Chem., 17, 227 (1978)

15. C. J. Persoons, P. E. J. Verwiel, F. J. Ritter, and W. J. Nooyen, J. Chem. Ecol., 8, 439 (1982).

16. Y. Shizuri, S. Yamaguchi, Y. Terada, and S. Yamamura, Tetrohedron Lett., 28, 1791 (1987).

17. Y. Shizuri, S. Yamaguchi, S. Yamamura, M. Ishihara, S. Ohba, Y. Saito, M. Niwa, Y. Terada, and M. Miyazaki, Tetrahodron Lett, 28, 3831 (1987).

18. T. L. Macdonald, C. M. Delahunty, and J. S. Sawyer, Heterocycles, 25, 305 (1987).

19. S. Takahashi, H.Takegawa, T. Takahashi, and T. Doi, J. Pesticide Sci., 13, 501 (1988).

20. Y. Shizuri, K. Matsunaga, K. Tamaki, S. Yamaguchi, and S. Yamamura, Tetrahedron Lett., 29,1971 (1988).

21. H. Hauptmann, G. Muhlbauer, and H. Sass, Tetrohedron Lett., 27, 6189 (1986).

22. C. Nishino, K. Kobayashi, M. Fukushima, M. Imanari, K. Nojima, and S. Kohno, Chem. Lett., 517 (1988).

23. Y. Shizuri, S. Yamaguchi, Y. Terada, and S. Yamamura, Tetrahedron Lett., 28, 1795 (1987).

24. Y. Shizuri, K. Matsunaga, and S. Yamamura, Tetrohedron Lett., 30, 3693 (1989).

25. S. Kuwahara and K. Mori, manuscript in preparation.

26. K. Mori and Y. Igarashi, Tetrahedron Lett., 30, 5145 (1989). In this communication we erroneously named 35 as PA 22-VIII. The correct code name as given by Persoons was PA 22-VII.

27. C. J. Persoons, "Structure Elucidation of Some Insect Pheromones" (doctoral dissertation), Wageningen, 1977. We thank Dr. Persoons for his kind gift of a copy of his dissertation.

28. H. Hauptmann, unpublished data. We were informed that he also noticed the thermal rearrangement of his periplanone-A to Persoons's by GC-MS.

29. S. Kuwahara and K. Mori, Tetrahedron Lett., in press. 